\title{
Review and extension of pressure drop models applied to Taylor flow regimes
}

\author{
Marc Mac Giolla Eain ${ }^{\mathrm{a}, *}$, Vanessa Egan ${ }^{\mathrm{a}}$, James Howard ${ }^{\mathrm{a}}$, Patrick Walsh ${ }^{\mathrm{b}}$, Edmond Walsh ${ }^{\mathrm{c}}$, Jeff Punch ${ }^{\mathrm{d}}$ \\ ${ }^{a}$ Stokes Institute, Mechanical Aeronautical \& Biomedical Engineering, University of Limerick, Limerick, Ireland \\ ${ }^{\mathrm{b}}$ Stokes Institute, Civil Engineering \&' Material Science Dept., University of Limerick, Limerick, Ireland \\ ' Osney Thermo - Fluids Laboratory, University of Oxford, England, United Kingdom \\ ${ }^{\mathrm{d}}$ CTVR, Stokes Institute, Mechanical Aeronautical \&' Biomedical Engineering, University of Limerick, Limerick, Ireland
}

\section{A R T I C L E I N F O}

\section{Article history:}

Received 24 February 2014

Received in revised form 29 August 2014

Accepted 1 September 2014

Available online 4 October 2014

\section{Keywords:}

Taylor flows

Slug flows

Pressure drop

Liquid-liquid

Liquid-gas

\begin{abstract}
A B S T R A C T
This paper investigates the pressure drop induced by both liquid-liquid and liquid-gas segmented Taylor flow regimes. A comprehensive experimental programme was completed using four different liquidliquid and three different liquid-gas combinations over dimensionless slug length, Reynolds and Capillary numbers that spanned several orders of magnitude. Comparisons between the liquid-liquid pressure drop data and the most referenced expressions in the literature highlighted their lack of robustness and demonstrated their inapplicability for use with most practical systems that incorporate liquid-liquid Taylor flow regimes. The experimental pressure drop values obtained for the liquid-gas flows agreed well with existing pressure drop correlations. Interpretation of the liquid-liquid data using liquid-gas models unearthed the existence of a threshold viscosity ratio. Above this threshold, experimental liquid-liquid data was found to agree well with existing liquid-gas models. Below this threshold, results showed that the dispersed slug velocity needed to be considered as the flows were subject to higher interfacial contributions and inertial effects. A modification is proposed to an existing liquid-gas pressure drop correlation. This proposed modification extends the applicability of the correlation to liquid-liquid flows, and furthermore extends the non-dimensional limits of the correlation.
\end{abstract}

(ㄷ) 2014 Elsevier Ltd. All rights reserved.

\section{Introduction}

At present there is considerable interest in the development of micro-electro mechanical systems (MEMS). MEMS can be thought of, in the most general form, as miniaturised mechanical and electro-mechanical devices and structures, and can vary from as little as $20 \mu \mathrm{m}$ up to $1 \mathrm{~mm}$ in size. MEMS have potential applications in a number of different fields including: biochemical and medical diagnostics, communications and sensing. The motivations in transferring existing technology to the small scale are numerous and include portability of devices and substantial savings in cost, for both the manufacturer and consumer. Although significant attention has been focused on the outputs of these small scale devices, little attention has been devoted to the complex fluid mechanics that govern the flow within them. Over the last decade, multiphase flows, be they liquid-liquid or liquid-gas, have become a prominent feature within these technologies that employ microfluidics.

\footnotetext{
* Corresponding author. Tel.: +353 61233619; fax: +353 61202393

E-mail address: marc.macgiollaeain@ul.ie (M. Mac Giolla Eain).
}

A multiphase flow regime is generated when two immiscible phases are pumped into a channel at a variety of flow rates. The generated flow regimes are the result of surface and body force interactions and include, but are not limited to: churn, annular, wavy, bubbly, slug and stratified. At the microscale, however, the dominance of surface forces over gravitational forces results in slug and droplet flow regimes being the most commonly encountered. Slug or Taylor flow regimes offer significantly enhanced levels of heat, (Walsh et al., 2010), and mass, (Ghaini et al., 2010), transfer over equivalent single phase flow systems. Consequently, they can be found in a diverse range of technologies including compact heat exchangers, (Muzychka et al., 2011), monolith catalysts, (Kreutzer et al., 2005b), and biomedical devices, (Gunther et al., 2004). The aforementioned technologies require a good understanding of the flow characteristics and hydrodynamics for their design and precise control.

The addition of a second immiscible phase to the flow results in a significant increase in the pressure drop relative to the single phase flow case. Pressure drop now becomes a key parameter as it affects flow rates, stability, sizing of the pumps and hence the overall cost of the system. There are, within the literature, a number of 
approaches that can be used to model the pressure drop of a multiphase flow regime. The simplest approach is the homogeneous flow model, which assumes the two phase flow mixture behaves as a pseudo fluid that has some average defined properties. The pressure drop is then calculated using single phase flow theory, (Salim et al., 2008). Another straightforward approach is that offered by the separated flow model. This modelling technique considers the contributions of both phases separately and predicts the resultant pressure drop to be the sum of their single phase contributions. However, both of these approaches are limited as neither accounts for the additional pressure drop associated with the interface that exists between the phases. (Lockhart and Martinelli, 1949) addressed this issue in their seminal paper and developed an empirical parameter to account for the effects of the interface. While this approach has garnered much popularity and use, it does not, however, distinguish between the different flow physics associated with the different flow regimes. Consequently, interfacial pressure drop predictions are, in general, an order of magnitude estimate, and hence are not acceptable for applications such as chemical processing or biomedical diagnostics.

The present work is focused upon the pressure drop associated with segmented Taylor or slug flow regimes in both liquid-liquid and liquid-gas flows. The pressure drop associated with a liquidgas Taylor flow regime has received significant attention in the literature and has been the subject of numerous numerical and experimental studies, (Awad and Muzychka, 2008; Kawahara et al., 2002; Saisorn and Wongwises, 2008; Triplett et al., 1999), however, the prediction of the pressure drop in liquid-liquid flows has received significantly less attention, (Gupta et al., 2013; Jovanovic et al., 2010; Tsaoulidis et al., 2013). The prediction is considerably more complicated due to the increased importance of the viscosity of the dispersed phase and depending on the viscosity ratio that exists between the phases, the resultant interfacial shear can have considerable effects on the overall pressure drop. The viscosity ratio between the liquid phases can vary greatly; and relative to water can range from approximately 0.3 , for tetradecane, up to 100 , for some silicone based oils, (Zheng and Ismagilov, 2005). Hence, the viscosity ratio between the phases can be comparable to that encountered in liquid-gas flow regimes. Consequently, provided a significant viscosity ratio exists between the phases, the approaches developed to predict the pressure drop of liquid-gas Taylor flow regimes should be applicable to liquidliquid Taylor flow regimes. However, the validity of this hypothesis has never been examined.

This work aims to address this gap in the literature and determine over which range of viscosity ratios certain models, developed for liquid-gas Taylor flow regimes, can be applied to liquid-liquid Taylor flow regimes. An experimental facility was built to measure the pressure drop in both liquid-gas and liquidliquid slug flow regimes flowing in small scale capillaries. The flow was analysed using an array of carrier fluids, while water and air were used as the discontinuous phase in all liquid-liquid and liquid-gas tests respectively. The variations in the physical properties resulted in measurements over a wide range of dimensionless parameters and extended greatly on the ranges previously presented in the literature. Comparisons are made between the experimental data and the most pertinent liquid-gas and liquid-liquid pressure drop models in the literature and a number of recommendations are made as to their use and applicability with the different flow regimes, be they liquid-gas or liquid-liquid.

\section{Theory}

This section highlights the relevant analytical and empirical expressions used to characterise a Taylor flow regime hydrodynamically. For fully developed Hagen-Poiseuille flow of a single phase fluid flowing in a capillary, the pressure drop is given by:

$\frac{\Delta P}{L}=\frac{16}{R e}\left(\frac{1}{2} \rho U^{2}\right) \frac{4}{D} \quad$ or $f R e=16$

where $\Delta P, L, R e, \rho, U, D$ and $f$ refer to the pressure drop, capillary length, Reynolds number, density, mean velocity of the flow, capillary diameter and friction factor, respectively. The introduction of a second phase into the capillary, to create a segmented flow regime, results in an increase in the pressure drop over the single phase flow case. Consequently, the total pressure drop in the channel may now be expressed as the sum of the contributions of the constituent phases, i.e. the single phase pressure drops of the different phases, and the additional pressure drop caused by the interface between the phases.

$\Delta P_{T}=\Delta P_{S}+\Delta P_{\text {Int }}$

where the subscripts $T, s$ and Int refer to the total, single phase and interfacial pressure drops.

\section{Liquid-gas models}

In a liquid-gas flow regime, the frictional losses in the gaseous bubbles are considered negligible when compared to those in the liquid phase, due to the large viscosity difference that exists between the phases, and as a result are not taken into account. Consequently, Eq. (2) can be rewritten as:

$f \operatorname{Re}_{T}=16+f \operatorname{Re}_{\text {Int }}$

The problem now turns to the determination of the interfacial component. A number of expressions have been developed to predict the interfacial pressure drop, with that of (Bretherton, 1960) being the most referenced in the literature and is presented here in Eq. (4).

$\Delta P_{\text {Int }}=7.16(3 C a)^{\frac{2}{3}} \frac{\sigma}{D}$

where $\mathrm{Ca}$ is the Capillary number and $\sigma$ is the interfacial tension between the respective phases. The above expression is a theoretical solution derived for the pressure drop caused by a single bubble in a Taylor flow regime. This expression was designed to be allencompassing, with changes in curvature due to the presence of the liquid film and the Laplace pressure term accounted for.

(Kreutzer et al., 2005a) examined the contribution of the interface both numerically and experimentally and determined the interfacial pressure drop to be a function of $L_{C}^{*}, \mathrm{Ca}$ and $\mathrm{Re}$. The authors developed the following expression to account for the pressure drop caused by the presence of the bubbles:

$f e_{B}=\frac{\alpha}{L_{C}^{*}}\left(\frac{R e}{C a}\right)^{\beta}$

where $\alpha$ and $\beta$ are constants whose values are presented in Table 1 and $L_{C}^{*}$ is the dimensionless carrier slug length, $L_{C}^{*}=L_{C} / D$. (Walsh et al., 2009) completed a rigorous experimental analysis of the problem and came to the same conclusion, that the interfacial pressure drop was a function of $L_{C}^{*}, C a$ and Re. However, (Walsh et al.,

Table 1

Range in constants used in the different liquid-gas Taylor flow models.

\begin{tabular}{lll}
\hline Model & $\alpha$ & $\beta$ \\
\hline Kreutzer et al. (2005a, 2005b) experimental & 2.72 & 0.33 \\
Kreutzer et al. (2005a, 2005b) numerical & 1.12 & 0.33 \\
Walsh et al. (2009) experimental & 1.92 & 0.33 \\
\hline
\end{tabular}


2009) found that a value of $\alpha=1.92$ provided a much better collapse of the data than those put forward by Kreutzer et al. (2005a).

More recently (Warnier et al., 2010) examined the contribution of the bubble to the total pressure drop in a Taylor flow regime. Similar to the works of (Aussillous and Quere, 2000), where the authors modified the expression of (Bretherton, 1960) and incorporated the data of (Taylor, 1960) to develop Taylor's Law, (Warnier et al., 2010) reworked Eq. (4), to incorporate Taylor's Law to account for the presence of the film and its effects on pressure drop. The resultant interfacial pressure drop expression is presented here in Eq. (6), where $\operatorname{Re}_{B}$ refers to the Reynolds number based on the liquid properties and the gas bubble velocity, and $A$ and $A_{B}$ refer to the capillary and bubble cross sectional areas, respectively.

$f e_{B}=\left(\frac{7.16\left(3^{\frac{2}{3}}\right)}{32} \frac{1}{L_{C}^{*}} \frac{A}{A_{B}} \frac{1}{C a^{\frac{1}{3}}+3.34 C a}\right)$

\section{Liquid-liquid models}

There are two categories of pressure drop models for liquidliquid Taylor flows, both of which have been developed by Kashid and Agar (2007) and Jovanovic et al. (2010). The first assumes that the dispersed phase makes an appreciable contribution to the total pressure drop, while the second assumes that the thin film that encapsulates the dispersed phase contributes significantly to the total pressure drop. In the former category the total pressure drop consists of three components: (1) the frictional pressure drop of the carrier phase, (2) the frictional pressure drop of the dispersed phase and (3) the interfacial component. Presented in Eqs. (7) and (8) are the models of (Kashid and Agar, 2007), Eq. (7), and (Jovanovic et al., 2010), Eq. (8) respectively.

$\Delta P_{T}=\frac{L}{L_{u}}\left(\frac{8 \mu_{C} U(1-\varepsilon) L}{R^{2}}+\frac{8 \mu_{D} U \varepsilon L}{R^{2}}\right)+\frac{2 L-L_{u}}{L_{u}}\left(\frac{2 \sigma}{D} \operatorname{Cos} \theta\right)$

$\Delta P_{T}=\frac{8 \mu_{C} U(1-\varepsilon) L}{R^{2}}+\frac{8 \mu_{D} U_{D} \varepsilon L}{(R-h)^{2}}+\frac{L}{L_{u}} 7.16(3 C a)^{\frac{2}{3}} \frac{\sigma}{D}$

where $L_{U}, \mu_{C}, \mu_{D}, R, \varepsilon, \theta, U_{D}$ and $h$ refer to the slug unit length, $\left(L_{U}=L_{C}+L_{D}\right)$, carrier phase viscosity, dispersed phase viscosity, capillary radius, dispersed phase fraction length. $\left(L_{D} / L_{U}\right)$, contact angle, dispersed phase velocity and film thickness, respectively.
Two models fall into the latter category of pressure drop models, again these have been developed by Kashid and Agar (2007) and Jovanovic et al. (2010). The model of (Kashid and Agar, 2007) assumes that the pressure drop induced by the film is the dominant pressure drop in a slug unit, hence the pressure drops associated with the interface and the carrier phase can be completely neglected. This approach, presented in Eq. (9), is based on the work of (Charles et al., 1961) who modelled the pressure drop of solid capsules flowing through a capillary and relates the pressure drop along the film to the single phase carrier pressure drop along the length of the capillary. To avoid confusion with the other pressure drop model developed by Kashid and Agar (2007), the model presented in Eq. (9) will be referred to henceforth as the capsule model.

$\Delta P_{T}=\left(\frac{\varepsilon}{1-k^{4}}\right) \Delta P_{S}$

where $k=(R-h) / R$. The model of (Jovanovic et al., 2010) assumes that the pressure drop caused by the non-negligible flow in the thin film that separates the aqueous slugs from the capillary wall is greater than that of the dispersed phase. Consequently, the middle term in Eq. (8) is replaced by the term presented in Eq. (10) and the total pressure drop in the channel can be modelled using the expression presented in Eq. (11). This model will be referred to as the moving film model.

$$
\begin{aligned}
\Delta P_{f} & =\frac{4 U_{D} L \varepsilon}{\frac{\left(R^{2}-(R-h)^{2}\right)}{\mu_{C}}+\frac{\left(0.5(R-h)^{2}\right)}{\mu_{D}}} \\
\Delta P_{T} & =\frac{8 \mu_{C} U(1-\varepsilon) L}{R^{2}}+\frac{4 U_{D} L \varepsilon}{\frac{\left(R^{2}-(R-h)^{2}\right)}{\mu_{C}}+\frac{\left(0.5(R-h)^{2}\right)}{\mu_{D}}}+\frac{L}{L_{u}} 7.16(3 C a)^{\frac{2}{3}} \frac{\sigma}{D}
\end{aligned}
$$

Experimental measurements will be compared with these models; both liquid-liquid and liquid-gas, in subsequent sections and their applicability will be assessed.

\section{Experimentation}

Experiments analysing the hydrodynamics of both liquid-gas and liquid-liquid slug flows in minichannel geometries were performed in the experimental facility shown schematically in Fig. 1. The facility was designed such that: (1) it allowed a steady slug flow regime to develop, whose inlet flow rates could be accurately controlled and measured and (2) allowed variations in pressure

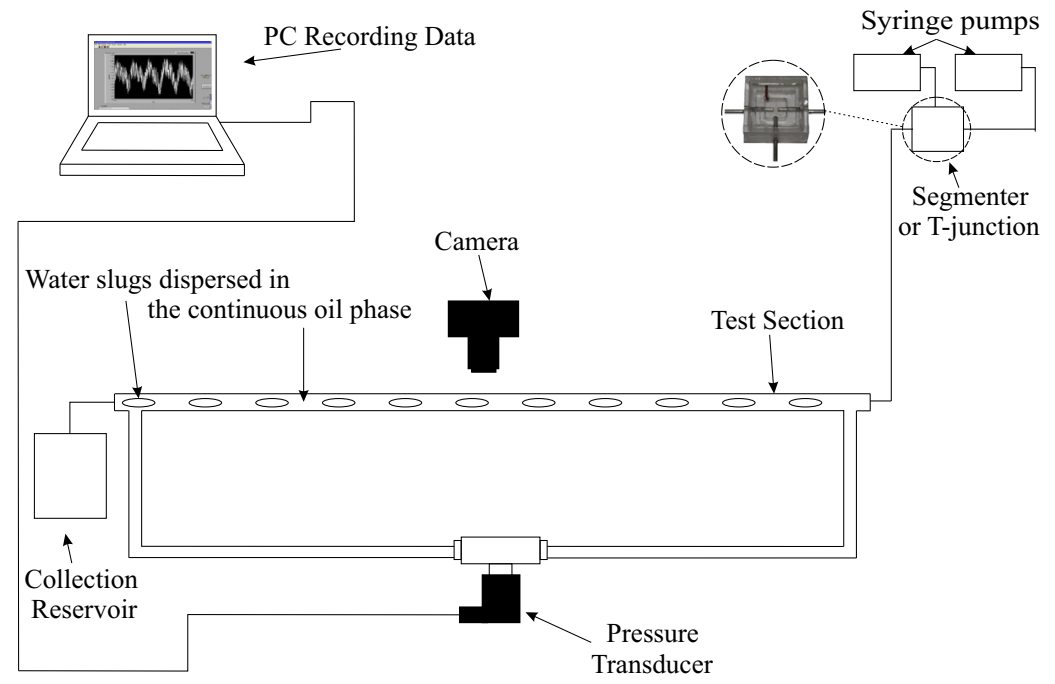

Fig. 1. Schematic illustration of experimental test facility. 
drop to be monitored and recorded over extended time periods to ensure steady state conditions had been achieved.

Two precision Harvard PhD 2000 programmable syringe pumps were used to set the volumetric flow rates. Separate syringe pumps allowed the relative flow rates, and hence slug lengths to be varied over a wide range. Both the liquid and gaseous phases were delivered by $100 \mathrm{ml}$ capacity Hamilton 1100TLL gas tight glass syringes. Glass syringes were used as they deliver steadier flow rates than those attainable from plastic syringes. The flows then merged to create well-ordered streams of liquid slugs separated by either a gaseous bubble or an aqueous droplet, depending on the flow regime under analysis. In the case of the liquid-gas flows, the phases combined in T-junctions of differing internal diameters, thus allowing a variety of slug and bubble lengths to be produced. While in the liquid-liquid analysis, T-junctions were used in conjunction with segmenters. Segmenters work by periodically creating and rupturing a liquid bridge between two opposing capillary tips and have been used previously by Curran et al. (2005) and Mac Giolla Eain et al. (2013) in liquid-liquid slug flow studies. Segmenters require a reservoir to be filled with a density matched oil to ensure that a buoyancy free environment exists for axisymmetric liquid bridging. Consequently, only the Pd5/water flows were generated using segmenters.

Once generated, the flow passed into the main test section. The pressure drop was measured in hard walled capillaries, stainless

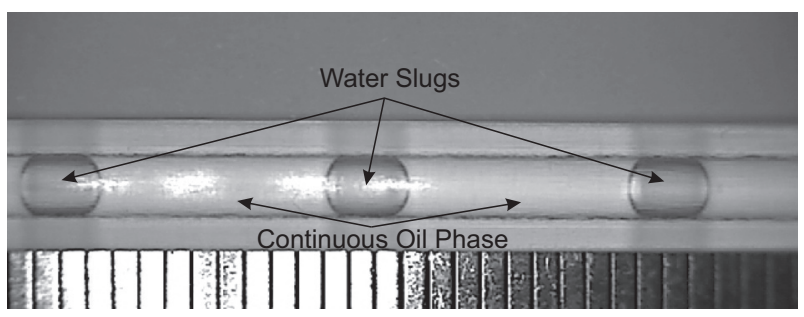

Fig. 2. Sample image of a liquid-liquid Taylor flow regime acquired using a CCD camera during experimentation. Continuous and dispersed slug lengths were determined by analysing images, such as this, in Matlab using the scale $(1 \mathrm{~mm}$ divisions) at the bottom.

Table 2

Thermophysical properties of the different experimental mediums, measured at room temperature and atmospheric pressure.

\begin{tabular}{lrrl}
\hline & $\begin{array}{c}\text { Density } \\
\rho\left(\mathrm{kg} / \mathrm{m}^{3}\right)\end{array}$ & $\begin{array}{l}\text { Viscosity } \\
\mu(\mathrm{kg} / \mathrm{m} \mathrm{s})\end{array}$ & $\begin{array}{l}\text { Interfacial tension } \\
\sigma(\mathrm{N} / \mathrm{m})\end{array}$ \\
\hline Air & 1.2 & $1.84 \times 10^{-5}$ & - \\
Water & 996.6 & $8.68 \times 10^{-4}$ & 0.073 \\
Pd5 & 911.8 & $3.58 \times 10^{-3}$ & 0.024 \\
Ethylene Glycerol & 1112.0 & $18.9 \times 10^{-3}$ & 0.048 \\
Pd5 & 911.8 & $3.58 \times 10^{-3}$ & 0.039 \\
FC40 & 1854.1 & $3.91 \times 10^{-3}$ & 0.051 \\
AR20 & 1142.2 & $20.91 \times 10^{-3}$ & 0.030 \\
Dodecane & 754.3 & $1.39 \times 10^{-3}$ & 0.052 \\
\hline
\end{tabular}

steel and FEP (fluorinated ethylene propylene) Teflon, that were horizontal in orientation and circular in cross section with a nominal internal diameter $1.59 \mathrm{~mm}$. The distance between the pressure tapings varied from $0.6 \mathrm{~m}$ to $2 \mathrm{~m}$ depending on the pressure range for each liquid used. A Druck PDCR 4170 series pressure transducer, manufacturer's accuracy of $0.1 \%$ of full scale deflection, with a range of $35 \mathrm{kPa}$ was used to allow for the range in pressure drops associated with the liquids of differing viscosities. Due to the long settling period required for steady state conditions to be achieved, the pressure transducer was connected to a PC which monitored the pressure variations over time. This was particularly pertinent for the liquid-gas flows as the gaseous phase is compressible and a longer settling period is required before steady state is achieved i.e. until constant slug and bubble lengths are observed. Consequently, a series of CCD cameras, placed at different locations, were used to monitor slug lengths during the course of the experimentation. A sample image showing a series of oil slugs punctuated by aqueous slugs is presented in Fig. 2. A custom code, developed in Matlab (version 2010a), was used to extract the average gaseous bubble, oil and aqueous slug lengths from a series of images for each test. The maximum variation in bubble and slug lengths was found to be less than $10 \%$.

Experiments were carried out using a selection of different liquids to provide a wide range in physical properties and hence Capillary and Reynolds numbers. In all liquid-gas experiments, air was used as the gaseous phase, while water was used as the dispersed phase in all liquid-liquid experiments. The carrier fluids examined were: water, AR20, FC40, Pd5, dodecane and ethylene glycol. The relevant physical properties for each of the different media are presented in Table 2, while the resultant dimensionless parameters of the experiments are presented in Table 3. The Reynolds and Capillary numbers span over several orders of magnitude, and the dimensionless slug, bubble and droplet lengths span over two orders of magnitude. These ranges are expected to reflect the ranges in non-dimensional parameters encountered in most micro/mini scale fluidic systems that incorporate either liquid-gas or liquid-liquid slug flow regimes.

\section{Results and discussion}

The following section presents and analyses experimental pressure drop measurements recorded for both liquid-liquid and liquid-gas Taylor flow regimes. The results are presented and discussed in two separate subsections that compare: the experimental liquid-liquid data with the most referenced liquid-liquid pressure drop models in the literature and both the liquid-liquid and liquid-gas data with the most pertinent liquid-gas pressure drop models in the literature.

\section{Liquid-liquid pressure drop models}

The following subsection presents a comparison of the experimental liquid-liquid pressure drop data with the models presented in Section 'Liquid-liquid models'.

Table 3

Resultant range in dimensionless parameters over which both liquid-gas and liquid-liquid studies were conducted.

\begin{tabular}{|c|c|c|c|c|}
\hline Liquid-gas study & $\operatorname{Re}$ & $\mathrm{Ca}$ & $L_{C}{ }^{*}$ & $L_{B}{ }^{*}$ \\
\hline Water/air & $56.75-567.59$ & $0.014-0.067$ & $1.17-11.34$ & $1.67-11.59$ \\
\hline Pd5/air & $17.90-59.67$ & $0.010-0.034$ & $2.86-46.83$ & $3.84-14.25$ \\
\hline Ethylene glycerol/air & $3.33-14.98$ & $0.015-0.067$ & $1.18-11.34$ & $1.67-11.59$ \\
\hline Liquid-liquid study & $\operatorname{Re}$ & $\mathrm{Ca}$ & $L_{C}^{*}$ & $L_{D}{ }^{*}$ \\
\hline Pd5/water & $12.61-44.19$ & $0.003-0.0098$ & $1.87-14.12$ & $1.65-8.10$ \\
\hline FC40/water & $18.75-97.48$ & $0.001-0.007$ & $1.09-9.94$ & $1.09-6.5$ \\
\hline AR20/water & $1.82-7.29$ & $0.014-0.052$ & $1.45-4.54$ & $1.13-1.33$ \\
\hline Dodecane/water & $1.45-86.91$ & $4.49 \times 10^{-5}-0.0027$ & $0.76-5.88$ & $1.05-5.12$ \\
\hline
\end{tabular}


(a)

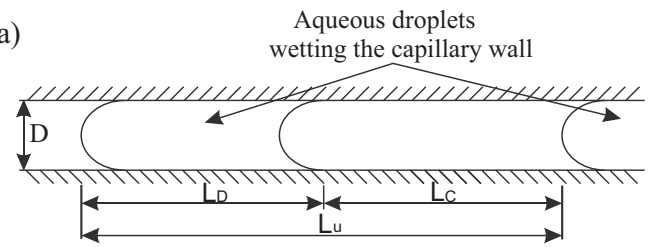

(b) Aqueous droplets suspended in a continuous

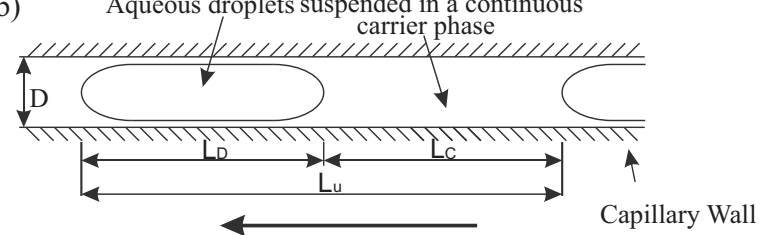

Flow direction

Fig. 3. Slug unit cell, consisting of a single continuous carrier slug and dispersed droplet, (a) slug unit cell without film and (b) slug unit cell with film.

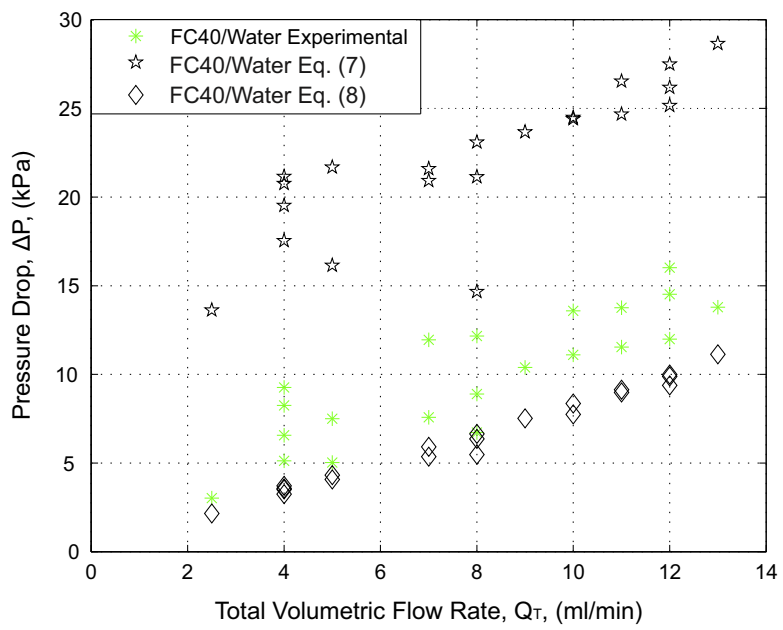

Fig. 4. Plot of the total volumetric flow rate against pressure drop for an FC40/water flow. Plot contains data points estimated using the models of Kashid and Agar (2007), Eq. (7) and Jovanovic et al. (2010), Eq. (8).

Fig. 4 is a plot of the total experimentally measured pressure drop against the total volumetric flow rate for an FC40/water flow. The flow rate and pressure drop range from $2.5 \mathrm{ml} / \mathrm{min}$ to $13 \mathrm{ml} / \mathrm{min}$ and $3 \mathrm{kPa}$ to $16 \mathrm{kPa}$ respectively. Included in the plot are data points estimated using the expressions from the first category of liquid-liquid pressure drop model, presented in Eqs. (7) and (8). It is evident from Fig. 4 that the model of (Kashid and Agar, 2007), Eq. (7), greatly overestimates the total pressure in the capillary, by approximately $10.1 \mathrm{kPa}$, (333.6\%), at the lower flow rates up to $14.5 \mathrm{kPa},(103.4 \%)$, at the higher flow rates. While the model of (Jovanovic et al., 2010), Eq. (8), underestimates the total pressure drop in the capillary, from $1 \mathrm{kPa}$ to $5 \mathrm{kPa}$,
(28.8-61.4\%), at the lower flow rates and up to $6 \mathrm{kPa},(41.4 \%)$, at the higher flow rates. Similar results were observed in the other liquid-liquid combinations examined in this study and the results are summarised in Table 4.

The model of (Kashid and Agar, 2007), Eq. (7), greatly overestimates the experimental data, in some cases the model data is almost double that of the experimental data, due to the method used to calculate the interfacial pressure drop. The model assumes no liquid film separates the aqueous slugs from the capillary wall, as shown in Fig. 3a). Consequently, the interfacial pressure drop is calculated at a constant contact angle and the contributions of the front and rear menisci are summed together. However, it has been shown by numerous investigators, (Han and Shikazono, 2009; Howard and Walsh, 2013; Olbricht and Kung, 1992; Taha and Cui, 2004), that a thin liquid film separates the dispersed slugs from the capillary wall, Fig. 3b). Therefore, the contact angles are substantially different from the dry wall case and the receding and advancing contact angles can only be assumed equal at very low velocities. Furthermore, due to the presence of the film, the rear cap of the slug assumes a profile that is the inverse to that of the front cap; hence the front cap has a positive contribution and the rear cap a negative contribution to the pressure drop. Accordingly, the contributions should be subtracted from one another rather than summed. In the model developed by Jovanovic et al. (2010), Eq. (8), the theoretical solution of Bretherton is used to determine the interfacial pressure drop. This expression underestimates the interfacial contribution as it assumes there are negligible inertial effects and that the film thickness is small compared to the capillary radius, $h / R<10^{-2}$. However, it has been shown by a number of authors, (Aussillous and Quere, 2000; Grimes et al., 2007; Mac Giolla Eain et al., 2013), that this assumption is invalid and that the theoretical solution of Bretherton breaks down at $\mathrm{Ca}<10^{-4}$ and $\mathrm{Ca}>10^{-1}$, and in cases where inertia is non-negligible, $R e>1$.

Fig. 5 is a plot of the same experimental FC40/water measurements as presented in Fig. 4. Included in the plot are data points estimated using the second category of pressure drop model, the capsule and moving film models presented in Eqs. (9) and (11) respectively. Unlike the data presented in Fig. 4, both models now underestimate the magnitude of the pressure drop, from $1 \mathrm{kPa}$ to $5 \mathrm{kPa},(28.9-61.6 \%)$, at the lower flow rates to between $5 \mathrm{kPa}$ and $8 \mathrm{kPa},(17.9-52.1 \%)$, at the higher flow rates. Similar results were observed in the other liquid-liquid combinations examined in this study and are summarised in Table 4. Both models put forward by Jovanovic et al. (2010) provide similar estimates of the experimental data. This result is unsurprising, as both models assume that the interface and the carrier phase make the greatest contribution to the overall pressure drop, between $90 \%$ and $95 \%$, and both models use the same expressions to estimate these contributions. However, as discussed previously, the model underestimates the total pressure drop as the theoretical Bretherton expression underestimates the interfacial component. Alternatively, the capsule model put forward by Kashid and Agar (2007) provides a better estimate than their previously discussed

Table 4

Summary of the differences between the experimental liquid-liquid data and data estimated using the most referenced liquid-liquid models.

\begin{tabular}{|c|c|c|c|c|}
\hline \multirow[t]{3}{*}{ Liquid-liquid combination } & \multicolumn{4}{|l|}{ Model } \\
\hline & \multicolumn{2}{|c|}{ Non-negligible dispersed phase effects } & \multicolumn{2}{|l|}{ Negligible dispersed phase effects } \\
\hline & Kashid and Agar, 2007, Eq. (7) & Jovanovic et al., 2010, Eq. (8) & Kashid and Agar, 2007, Eq. (9) & Jovanovic et al., 2010, Eq. (11) \\
\hline Pd5/water & $154.9-417.1 \%$ & $3.7-60.8 \%$ & $2.1-48.9 \%$ & $3.5-60.9 \%$ \\
\hline Dodecane/water & $79.4-518.1 \%$ & $85.7-307.3 \%$ & $18.2-74.7 \%$ & $85.5-306.7 \%$ \\
\hline FC40/water & $68.6-365.3 \%$ & $17.6-61.5 \%$ & $5.3-54.9 \%$ & $17.9-61.6 \%$ \\
\hline AR20/water & $76.1-420.8 \%$ & $2.5-31.1 \%$ & $2.5-28.7 \%$ & $2.5-30.9 \%$ \\
\hline
\end{tabular}




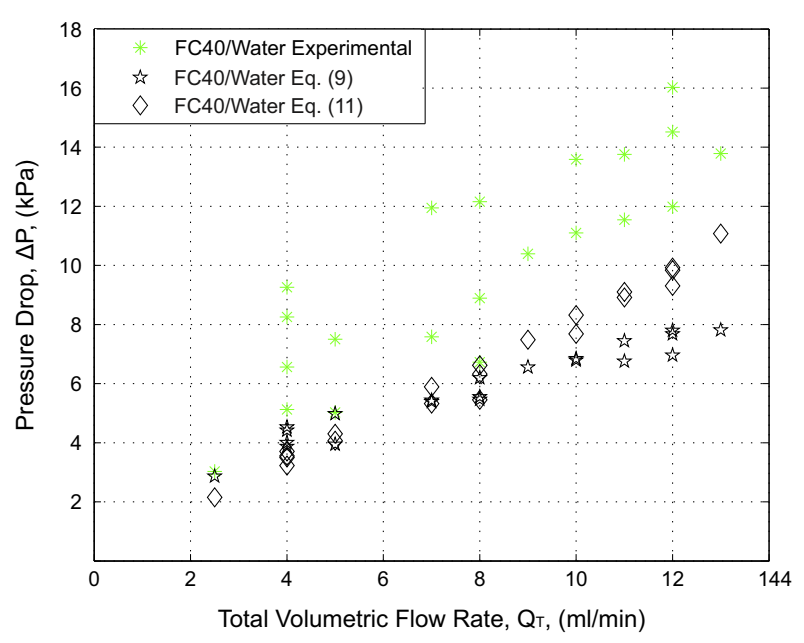

Fig. 5. Plot of flow rate against pressure drop for an FC40/water flow. The plot contains experimentally measured data points and data points estimated using the models of Kashid and Agar (2007), Eq. (9) and Jovanovic et al. (2010), Eq. (11). In this case the models assume that liquid film makes a significant contribution to the total pressure drop.

model, presented in Eq. (7). Nonetheless, it still provides poor agreement with the experimental data, underestimating the magnitude of the pressure drop from between $0.8 \mathrm{kPa}$ and $6.7 \mathrm{kPa}$, (17.9-61.6\%), respectively. This model, based on that of (Charles et al., 1961), assumes that the flow in the aqueous droplets is analogous to that in a solid capsule. This implies that there is no circulation of flow within the aqueous droplets and a constant cap shape exists. This is not the case for liquid-liquid Taylor flows, where the circulation of flow within the slugs has been documented in numerous $\mu$-PIV studies, such as those by Miessner et al. (2008), Malsch et al. (2008), and King et al. (2007). The capsule model is too simplistic, does not account for the hydrodynamics of the flow and, ultimately, underpredicts the magnitude of the pressure drop.

In summary, none of the discussed models provides an accurate estimate, within $\pm 15 \%$, of the experimentally measured pressure drop data. The major failing of these models lies in the methods used to calculate the interfacial pressure drop. The expressions used are very limited in terms of their applicability, to cases where there are negligible inertial effects or the dispersed phases wet the capillary walls, and as a result are not suitable for use in the design of most systems that incorporate liquid-liquid Taylor flow regimes. Numerical modelling of liquid-gas Taylor flows by Fujioka and Grotberg (2005), Heil (2001), and Ratulowski and Chang (1989) has shown that the flow structure around the dispersed slugs is influenced by inertia, and this influence on the interfacial pressure drop has been confirmed experimentally by Kreutzer et al. (2005a), Walsh et al. (2009), and Warnier et al. (2010). Consequently, the interfacial pressure drop in a liquidliquid Taylor flow regime can also be expected to be influenced by inertia, thus, liquid-gas models should provide some guidelines towards modelling the pressure drop experienced by liquid-liquid flows.

\section{Liquid-gas pressure drop models}

The following subsection presents a comparison of the experimental liquid-liquid and liquid-gas pressure drop data with the most pertinent liquid-gas pressure drop correlations from the literature, presented previously in Section 'Liquid-gas models'. In numerous microfluidic devices that incorporate liquid-liquid
Table 5

Viscosity differences between the different liquid-gas and liquidliquid combinations examined in the present study.

\begin{tabular}{ll}
\hline Fluid combinations & Viscosity ratio $\left(\mu_{C} / \mu_{D}\right)$ \\
\hline Water/air & 54 \\
Pd5/air & 190 \\
Ethylene glycerol/air & 953 \\
Pd5/water & 4.1 \\
Dodecane/water & 1.5 \\
FC40/water & 4.5 \\
AR20/water & 23 \\
\hline
\end{tabular}

Taylor flows, such as those presented by Cramer et al. (2004) and Sugiura et al. (2004), the viscosity difference between the liquid phases can be similar in magnitude to that encountered in liquid-gas flow regimes. Presented in Table 5 are the viscosity ratios, viscosity of the carrier phase to that of the dispersed phase, of the different liquid-liquid and liquid-gas combinations examined in the present study. It is evident from Table 5 that the viscosity difference that exists between the liquid-gas flows is quite substantial, with the carrier phase being 54-953 times more viscous, while the difference between the liquid-liquid flows is much more modest, with the oils being 1.6-23 times more viscous than water. Nonetheless, in a previous study by Salim et al. (2008), where the carrier phase was approximately 30 times more viscous than the dispersed phase, the authors found good agreement between their experimental data and data modelled using liquid-gas models.

There are numerous liquid-gas Taylor flow models in the literature. Previous studies in this area by Horvath et al. (1973), Walsh et al. (2010), and Howard et al. (2011) have shown slug length to be an important parameter, significantly influencing the resultant pressure drop, heat and mass transfer rates. Nonetheless, to the best of the authors' knowledge, there are only three models in the literature that account for the effects of slug length on pressure drop. These models have been presented in Section 'Liquid-gas models'. (Kreutzer et al., 2005a) developed two models, one based on experimental data and the other on numerical modelling. Both works found that the interfacial pressure drop could be scaled using the same dimensionless groups, presented in Eq. (5), however, the value of the constant $\alpha$ varied. Examining the problem experimentally over a much greater range, $1.58 \leqslant R e \leqslant 1024$ and $0.002 \leqslant C a \leqslant 0.2$, and incorporating the data of (Kreutzer et al., 2005a; Walsh et al., 2009) found that a value of $\alpha=1.92$, coincidently this is approximately the average of the simulation and experimental values of Kreutzer, provided much better agreement with the data. Consequently, the models of (Kreutzer et al., 2005a) will not be considered further in the analysis.

Fig. 6 present a direct comparison of the experimental friction factor - Reynolds number products, $\left(f_{\text {expTotal }} R e\right)_{\mathrm{TP}}$, and those calculated using the models of (Walsh et al., 2009), Eq. (5). The experimental friction factors were calculated using the following expression:

$f_{\text {expTotal }}=\left(\frac{\frac{P}{L}}{\left(\frac{1}{2} \rho_{C} U^{2}\right)\left(\frac{4}{D}\right)\left(\frac{Q_{C}}{Q_{T}}\right)+\left(\frac{1}{2} \rho_{D} U^{2}\right)\left(\frac{4}{D}\right)\left(\frac{Q_{D}}{Q_{T}}\right)}\right)$

To highlight the accuracy of the model, lines representing $\pm 20 \%$ deviations from parity are included in the plot. Starting with the liquid-liquid data, the Walsh model shows high levels of scatter in the Dodecane/water data. This indicates that the model is unsuitable for flows where the viscosities of the constituent phases are almost equal, in this case 1.6. As the viscosity ratio increases to 4.1 , the model begins to over predict the Pd5/water data and a further increase in viscosity ratio to 4.5 results in an improved model 


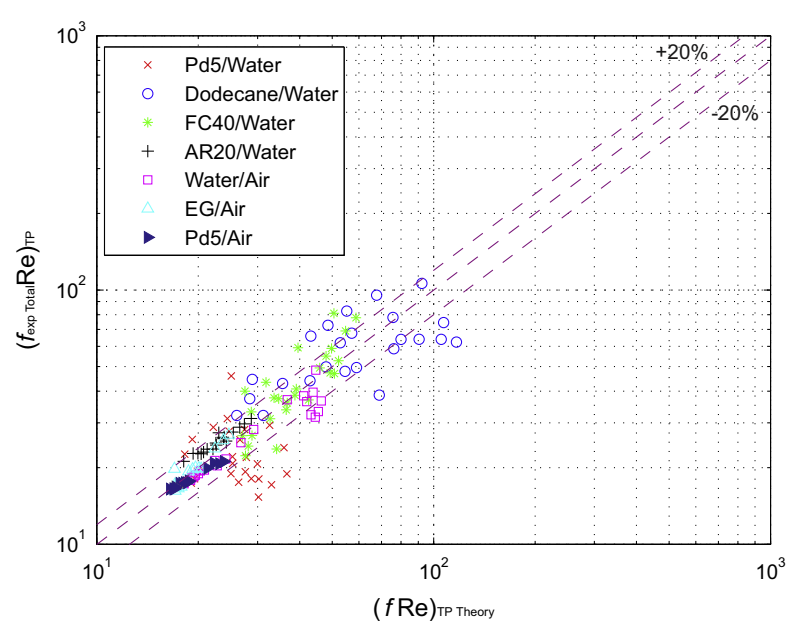

Fig. 6. Experimental pressure drop, presented as $\left(f_{\operatorname{expTotal}} R e\right)_{\mathrm{TP}}$, as described by Eq. (12), plotted against $(f R e)_{\mathrm{TP}}$ Theory, where the latter is calculated using the model of Walsh et al. (2009).

performance. This improvement in model performance continues as the viscosity ratio increases, with all of the AR20/water data falling inside the $\pm 20 \%$ bandwidth. This highlights the applicability of this model for high viscosity ratio flows. Given the range in $\mathrm{Ca}$ of the AR20/water flows, $0.014 \leqslant C a \leqslant 0.052$, falls perfectly within that of the Walsh model, $0.002 \leqslant C a \leqslant 0.2$, this result is unsurprising. The model proposed by Walsh et al. (2010) consists of a linear superposition of the single phase Poiseuille flow limit and a semi empirically derived interfacial flow limit and was developed using an addition of asymptotes approach. The transition from a Poiseuille to interfacially dominated flow occurs when $L_{\mathrm{C}}^{*}(\mathrm{Ca} / \mathrm{Re})^{0.33} \sim 0.12$. Thus, when $L_{\mathrm{C}}^{*}(\mathrm{Ca} / \mathrm{Re})^{0.33} \ll 0.12$, the pressure drop in the flow is dominated by the interface. While the opposite is true when $L_{C}^{*}(\mathrm{Ca} / \mathrm{Re})^{0.33} \ll 0.12$, the pressure drop in the flow is characteristically Poiseuille flow.

This is illustrated in Fig. 7, where the experimental liquid-liquid pressure drop data is plotted against the Walsh model. Included in the plot is the liquid-gas pressure drop data, consisting of Pd5/air, ethylene glycol/air and water/air measurements. At $L_{C}^{*}(\mathrm{Ca} / \mathrm{Re})^{0.33}>0.12$, the flows are subject to reduced interfacial effects and the slugs are of sufficient length for the pressure drop in the capillary to be characteristically Poiseuille. Due to its viscous nature, the data in this region is almost exclusively populated by the AR20/water flows. Similar to Fig. 6, excellent agreement is observed between the experimental data and the model. Thus highlighting the applicability of the Walsh model to flows where a significant viscosity difference, $\mu_{C} / \mu_{D}>4.5$, exists between the liquid phases in a liquid-liquid Taylor flow regime. With regards to the liquid-gas flows, there appears to be reasonable agreement between the experimental data and the Walsh model, for all liquid phases. However, some deviations can be seen between the model and the water-air flows for high interfacial contributions with increased Re values. In Fig. 7, results appear to scatter about the correlation. Very short slug lengths demonstrate the most significant deviations from the model as they have the greatest interfacial contribution, as described by $L_{C}^{*}(\mathrm{Ca} / \mathrm{Re})^{0.33}<0.12$. In this region, both the liquid-liquid and liquid-gas data sets are subject to increased inertial effects. (Kreutzer et al., 2005a) stated that Eq. (5) was only applicable for Re of order 100 , while the interfacial contribution calculated using the Bretherton model, Eq. (4), should only be used for $R e$ of order 1 . The data sets in this region, $L_{C}^{*}(\mathrm{Ca} / \mathrm{Re})^{0.33}<0.12$, have $R e$ ranging from 1.45 to 97.48 . Consequently, the poor performance of the Walsh model at these intermediate Re values is unsurprising.

A similar observation was made by Warnier et al. (2010), who noted that there was a dependence of the interfacial pressure on $R e$ at these intermediate Re values. The authors developed a new pressure drop model, presented in Eq. (6), which incorporated the works of (Aussillous and Quere, 2000) to account for the effects of intermediate $R e$ values. Fig. 8 presents the experimental data, $\left(f_{\exp }{ }_{\text {Total }} R e\right)_{\text {TP, }}$, plotted against data points calculated using the

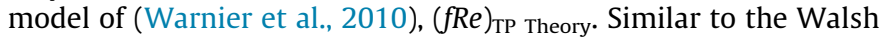
model, Figs. 6 and 7, there is excellent agreement between the Warnier model and the AR20/water and liquid-gas data sets. The Warnier model takes into account the increased dispersed slug velocity when determining the interfacial pressure drop and exhibits better agreement with changes in Re compared to the Walsh model. The Warnier model was developed over the following ranges in non-dimensional parameters: $41 \leqslant R e \leqslant 159$, $2.3 \times 10^{-3} \leqslant C a \leqslant 8.8 \times 10^{-3}, 3 \leqslant L_{C}^{*} \leqslant 23.95$ and $1.77 \leqslant L_{D}^{*} \leqslant 3.56$. The experimental liquid-gas data presented in this work significantly extends these ranges, $3.3 \leqslant R e \leqslant 567.59,0.01 \leqslant C a \leqslant 0.067$, $1.17 \leqslant L_{C}^{*} \leqslant 46.83$ and $1.67 \leqslant L_{D}^{*} \leqslant 14.25$ and validates the model for liquid phases other than water. Although the Warnier model provides an improved approximation of the liquid-liquid data, there are still a number of the Pd5/water, Dodecane/water and FC40/water data points outside of the $\pm 20 \%$ bandwidths.

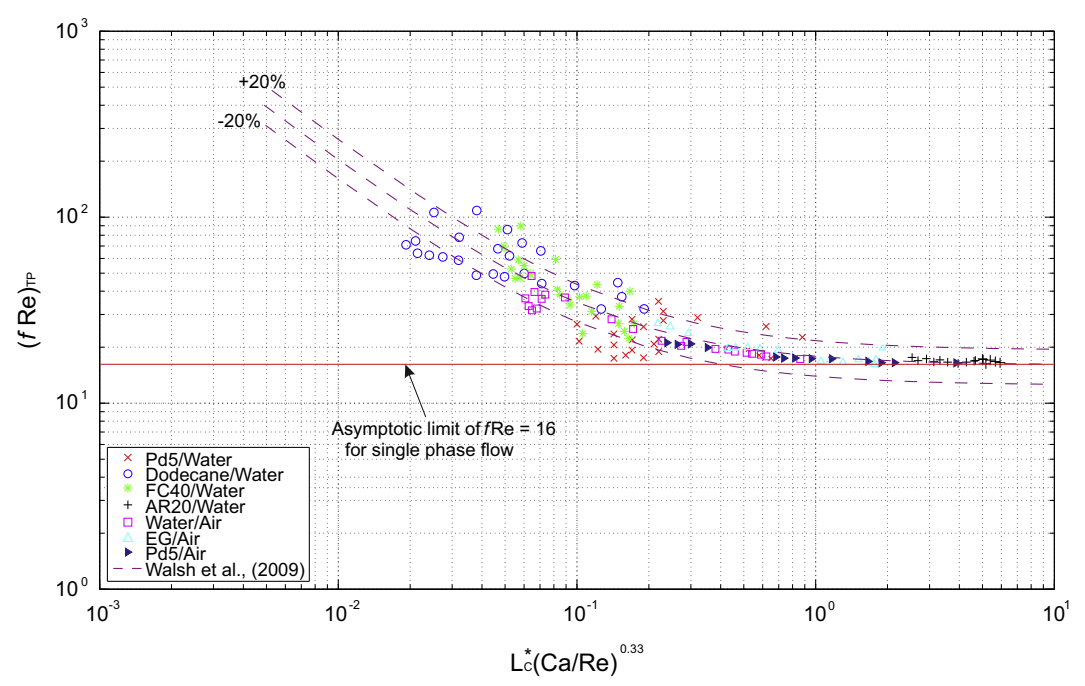

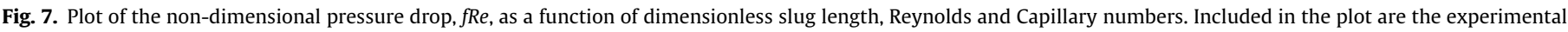
liquid-liquid and liquid-gas data, and the expression of Walsh et al. (2009). 


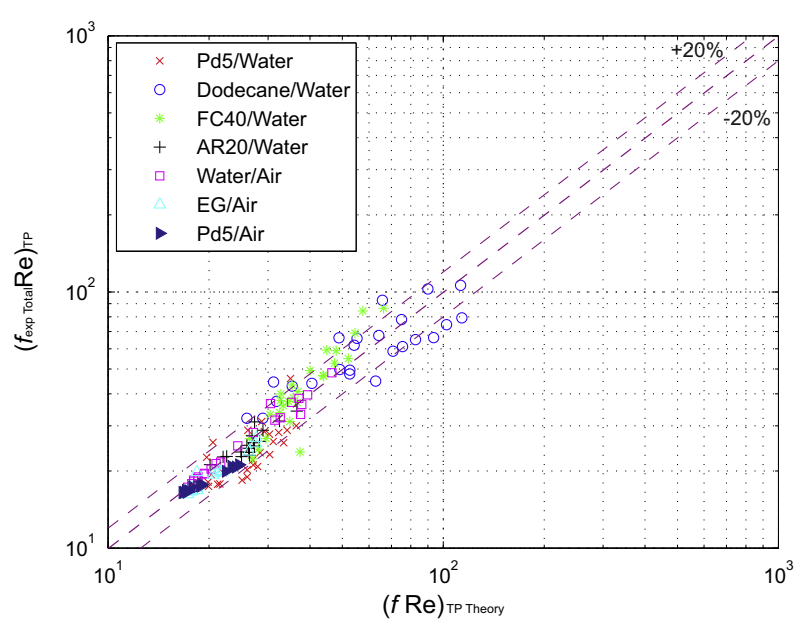

Fig. 8. Experimental pressure drop, presented as $\left(f_{\text {expTotal }} R e\right)_{\mathrm{TP}}$, as described by Eq. (12), plotted against $(f R e)_{\text {TP Theory, where the latter is calculated using the model }}$ of Warnier et al. (2010).

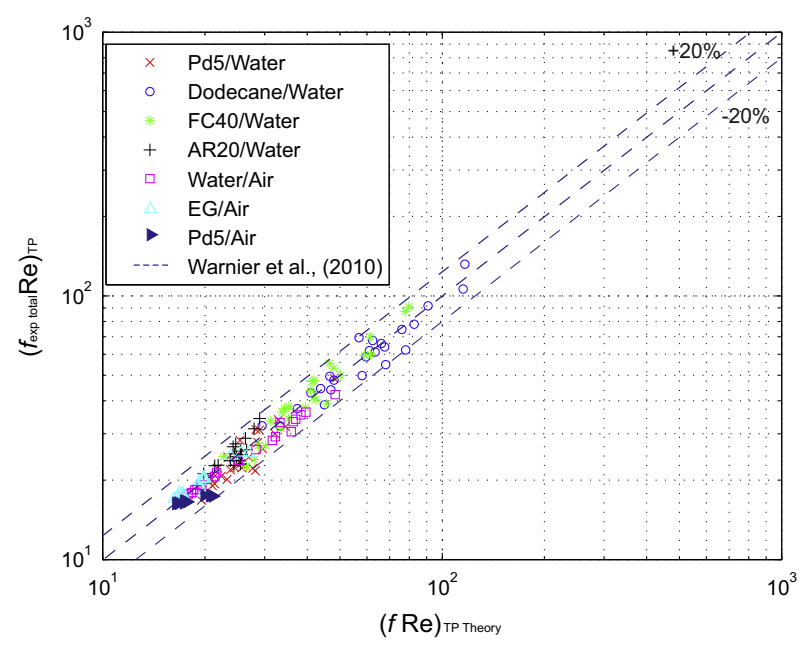

Fig. 9. Experimental pressure drop - presented as $\left(f_{\text {expTotal }} R e\right)_{\mathrm{TP}}$ and calculated using Eq. (12) - plotted against $(f R e)_{\text {TP }}$ Theory. The theoretical pressure drops were calculated using Eq. (3), where the interfacial component was calculated using a modified Warnier model, presented in Eq. (13).

A modified form of the interfacial component of the Warnier model is presented in Eq. (13). The interfacial pressure drop is normalised by the volume fraction of the capillary occupied by the carrier phase, $1-\alpha$, where $\alpha$ is the volume fraction of the dispersed phase and can be calculated using Eq. (14).

$f \operatorname{Re}_{B}=\left(\frac{8.16\left(3^{\frac{2}{3}}\right)}{32} \frac{1}{L_{C}^{*}} \frac{A}{A_{B}} \frac{1}{C a^{\frac{1}{3}}+3.34 C a}(1-\propto)^{1 / 3}\right)$

$\propto=\frac{Q_{D}}{Q_{T}}$

Eq. (13) implies that changes in the frictional pressure drop induced by the interface, $f R e_{B}$, due to the varying volumetric presence of the dispersed slugs, is negated by normalising $f R e_{B}$ by the volumetric channel fraction occupied by the carrier phase, $1-\alpha$. The curvature parameter in the original Warnier model, Eq. (6), is stated as 7.16 and is based on the work of (Bretherton, 1960). However, for the present work a value of 8.16 was found to provide a better approximation of the experimental data. Fig. 9 evaluates the performance of the modified Warnier model, where plotted on the abscissa are the theoretical fRe values and plotted on the ordinate are the experimental results. The outlying liquid-liquid data from Fig. 8 now resides within the $\pm 20 \%$ bandwidths and thus further extends the applicability of the Warnier model to the following ranges in non-dimensional parameters: $1.45 \leqslant R e \leqslant 567.59, \quad 4.5 \times 10^{-5} \leqslant C a \leqslant 0.067, \quad 0.76 \leqslant L_{C}^{*} \leqslant 46.83$ and $1.05 \leqslant L_{D}^{*} \leqslant 14.25$ and liquid-liquid flows. The excellent agreement between both the liquid-liquid and liquid-gas pressure drop data and this modified Warnier model implies that the contribution of the dispersed phase, be it liquid or gas, is negligible and can be completely neglected from consideration.

\section{Conclusions}

The current study reported novel experimental measurements examining the hydrodynamics of both liquid-liquid and liquidgas Taylor flow regimes. Experiments were conducted using four different liquid-liquid and three different liquid-gas combinations over dimensionless Reynolds and Capillary number ranges that spanned several orders of magnitude. Comparisons were made between the experimental liquid-liquid data and data points estimated using the most referenced correlations in the literature. Poor agreement was found between the data sets and this was attributed to: the assumptions used to develop the models being inapplicable to the type of flow regime under analysis, for example the flow in the dispersed phase being analogous to that in a solid capsule, and the methods used to calculate the contribution of the interface, where the expressions used are limited in applicability to very specific flow conditions.

Existing pressure drop correlations proposed by Walsh et al. (2009) and Warnier et al. (2010) have been shown to predict the pressure drop associated with liquid-gas slug flows in minichannels. The model proposed by Warnier et al. (2010) is observed to predict pressure drop with changes in Reynolds numbers, a limitation of previous models, however, it requires gas bubble velocity and this is typically not measured in practical applications. Experimental results agreed well with this model and significantly extended its non-dimensional limits to: $3.3 \leqslant R e \leqslant 567.59$ and $C a \leqslant 0.067$. Interpretation of the experimental liquid-liquid data using these models revealed the existence of a threshold viscosity ratio, 4.5. Above this threshold, the model developed by Walsh et al. (2010) provides excellent agreement with the experimental data, to within $\pm 15 \%$. Below this threshold, a modification is proposed to the interfacial component of the Warnier model. This modified Warnier model fits the remaining experimental data, to within $\pm 20 \%$, and further extends the applicability of the model to: $1.45 \leqslant \operatorname{Re} \leqslant 567.59,4.5 \times 10^{-5} \leqslant C a \leqslant 0.067,0.76 \leqslant L_{C}^{*} \leqslant 46.83$ and $1.05 \leqslant L_{D}^{*} \leqslant 14.25$ and liquid-liquid flows.

Overall, the findings reported herein provide some novel insights into the pressure drop experienced in both liquid-liquid and liquid-gas Taylor flows and should aid in the accurate prediction of pressure drop for future application based works.

\section{Acknowledgements}

The authors gratefully acknowledge the financial support of the Irish Research Council through the Embark Initiative. The involvement of Jeff Punch is supported by Science Foundation Ireland under Grant No. 10/CE/I1883.

\section{References}

Aussillous, P., Quere, D., 2000. Quick deposition of a fluid on the wall of a tube. Phys Fluids 12, 2367-2371.

Awad, M.M., Muzychka, Y., 2008. Effective property models for homogeneous two phase flows. Exp. Thermal Fluid Sci. 33, 106-113.

Bretherton, F.P., 1960. The motion of long bubbles in tubes. J. Fluid Mech. 10, 166-188. 
Charles, M.E., Govier, G.W., Hodgson, G.W., 1961. The horizontal pieline flow of equal density oil-water mixtures. Can. J. Chem. Eng. 39, 27-36.

Cramer, C., Fischer, P., Windhab, E., 2004. Drop formation in a Co-flowing ambient fluid. Chem. Eng. Sci. 59, 3045-3058.

Curran, K., Colin, S., Baldas, L., Davies, M., 2005. Liquid bridge instability applied to microfluidics. Microfluid. Nanofluid. 1, 336-345.

Fujioka, H., Grotberg, J.B., 2005. The steady propagation of surfactant laden liquid plug in a two-dimensional channel. Phys. Fluids 8, 1-17.

Ghaini, A., Kashid, M.N., Agar, D.W., 2010. Effective interfacial area for mass transfer in the liquid-liquid slug flow microreactors. Chem. Eng. Process. 49, 358-366.

Grimes, R., King, C., Walsh, E., 2007. Film thickness for two phase flow in a microchannel. Adv. Appl. Fluid Mech. 2, 59-70.

Gunther, A., Khan, S.A., Thalmann, M., Trachsel, F., Jensen, K.F., 2004. Transport and reaction in microscale segmented gas-liquid flow. Lab Chip 4, 278-286.

Gupta, R., Leung, S.S.Y., Manica, R., Fletcher, D.F., 2013. Hydrodynamics of liquidliquid Taylor flow in microchannels. Chem. Eng. Sci. 92.

Han, Y., Shikazono, N., 2009. Measurement of the liquid film thickness in micro tube slug flow. Int. J. Heat Fluid Flow 30, 842-853.

Heil, M., 2001. Finite Reynolds number effects in the Bretherton problem. Phys. Fluids 13, 2517-2521.

Horvath, C., Solomon, B.A., Engasser, J.M., 1973. Measurement of radial transport in slug flow using enzyme tubes. Ind. Eng. Chem. Fundam. 12, 431-439.

Howard, J.A., Walsh, P.A., 2013. Review and extensions to film thickness and bubble drift velocity prediction methods in laminar Taylor or slug flows. Int. J. Multiph. Flow 55, 32-42.

Howard, J.A., Walsh, P.A., Walsh, E.J., 2011. Prandtl and capillary effects on heat transfer performance within laminar liquid-gas slug flows. Int. J. Heat Mass Transf. 54, 4752-4761.

Jovanovic, J., Zhou, W., Rebrov, E.V., Nijhuis, T.A., 2010. Liquid-liquid slug flow: hydrodynamics and pressure drop. Chem. Eng. Sci. 66, 42-54.

Kashid, M.N., Agar, D.W., 2007. Hydrodynamics of liquid-liquid slug flow capillary microreactor: flow regimes, slug size and pressure drop. Chem. Eng. J. 131 $1-13$.

Kawahara, A., Chung, P.M.Y., Kawaji, M., 2002. Investigation of two phase flow pattern, void fraction and pressure drop in a microchannel. Int. J. Multiph. Flow 28, 1411-1435.

King, C., Walsh, E.J., Grimes, R., 2007. PIV measurements of flow within plugs in a microchannel. Microfluid. Nanofluid. 3, 463-472.

Kreutzer, M.T., Kaptejin, F., Moulijn, J.A., Heiszwolf, J.J., 2005a. Intertial and interfacial effects on pressure drop of Taylor flow in capillaries. AIChE J. 51, 2428-2440.

Kreutzer, M.T., Kaptejin, F., Moulijn, J.A., Heiszwolf, J.J., 2005b. Multiphase monolith reactors: chemical reaction engineering of segmented flow in microchannels. Chem. Eng. Sci. 60, 5895-5916.

Lockhart, R.W., Martinelli, R.C., 1949. Proposed correlation of data for isothermal two-phase, two-component flow in pipes. Chem. Eng. Prog. 45, 39-48.
Mac Giolla Eain, M., Egan, V., Punch, J., 2013. Film thickness measurements of liquid-liquid slug flow regimes. Int. J. Heat Fluid Flow 44, 515-523.

Malsch, D., Kielpinski, M., Merthan, R., Albert, J., Mayer, G., Khler, J.M., 2008. MicroPIV analysis of Taylor flow in microchannels. Chem. Eng. J. 135, 166-172.

Miessner, U., Lindken, R., Westerweel, J., 2008. 3D-velocity measurements in microscopic two phase flows by means of micro-PIV. In: 14th International Symposium on Applications of Laser Techniques to Fluid Mechanics.

Muzychka, Y.S., Walsh, E.J., Walsh, P.A., 2011. Heat transfer enhancement using laminar gas-liquid slug flow regimes . ASME J. Heat Trans. 133, 1-9.

Olbricht, W.L., Kung, D.M., 1992. The deformation and breakup of liquid drops in low Reynolds number flow through a capillary. Phys. Fluids 4, 1347-1354.

Ratulowski, J., Chang, H., 1989. Transport of gas bubbles in capillaries. Phys. Fluids $10,1642-1655$.

Saisorn, S., Wongwises, S., 2008. Flow Pattern. Void fraction and pressure drop of two phase air-water flow in a horizontal circular micro-channel experimental thermal and fluid science $32,748-760$.

Salim, A., Fourar, M., Pironon, J., Sausse, J., 2008. Oil-Water two-phase flow in microchannels: flow patterns and pressure drop measurements. Can. J. Chem. Eng. 86, 978-988.

Sugiura, S., Nakajima, M., Yamamoto, K., Iwamoto, S., Oda, T., Satake, M., Seki, M., 2004. Preparation characteristics of water-in-oil-in-water multiple emulsions using microchannel emulsification. J. Colloid Interface Sci. 70, 221-228.

Taha, T., Cui, Z.F., 2004. Hydrodynamics of slug flow inside capillaries. Chem. Eng. Sci. 59, 1181-1190.

Taylor, G., 1960. Deposition of a viscous fluid on the wall of a tube. J. Fluid Mech. 10, $161-165$.

Triplett, K.A., Ghiaasiaan, S.M., Abdel-Khalik, S.I., LeMouel, A., McCord, B.N., 1999. Gas-liquid two-phase flow in microchannels, Part 2: Void fraction and pressure drop. Int. J. Multiph. Flow 25, 395-410.

Tsaoulidis, D., Dore, V., Angeli, P., Plechkova, N.V., Seddon, K.R., 2013. Flow patterns and pressure drop of ionic liquid-water two-phase flows in microchannels. Int. J. Multiph. Flow 54, 1-10.

Walsh, E., Muzychka, Y., Walsh, P., Egan, V., Punch, J., 2009. Pressure drop in two phase slug/bubble flows in mini scale capillaries. Int. J. Multiph. Flow 35, 879884.

Walsh, P.A., Walsh, E.J., Muzychka, Y.S., 2010. Heat transfer model for gas-liquid slug flows under constant heat flux. Int. J. Heat Mass Transf. 53, 3193-3201.

Warnier, M.J.F., deCroon, H.J.M., Rebrov, E.V., Schouten, J.C., 2010. Pressure drop of gas-liquid Taylor flow in round microcapillaries for low to intermediate reynolds numbers. Microfluid. Nanofluid. 8, 33-45.

Zheng, B., Ismagilov, R., 2005. A microfluidic approach for screening submicroliter volumes against multiple reagents by using performed arrays of nanoliter plugs in three phase liquid/liquid/gas flow. Angew. Chem. Int. Ed. 44, 2520-2523. 